\title{
Mouse Nerve Growth Factor Facilitates the Growth of Interspinal Schwannoma Cells by Activating NGF Receptors
}

\author{
Shu Yi Liu, ${ }^{1} *$ Sheng Ze Liu, ${ }^{2, *}$ Yu Li, ${ }^{3}$ Shi Chen ${ }^{2}$ \\ School of Clinical Medicine, Xi'an Medical University, Xi'an, China \\ Department of Neurosurgery, ${ }^{2}$ Fuzhou Second Affiliated Hospital of Xiamen University, Fuzhou, China \\ Department of Otolaryngology, ${ }^{3}$ Second Affiliated Hospital of Xian Medical University, Xian, China
}

Objective : Nerve growth factor (NGF) is a member of the neurotrophic factor family and plays a vital role in the physiological processes of organisms, especially in the nervous system. Many recent studies have reported that NGF is also involved in the regulation of tumourigenesis by either promoting or suppressing tumor growth, which depends on the location and type of tumor. However, little is known regarding the effect of NGF on interspinal schwannoma (IS). In the present study, we aimed to explored whether mouse nerve growth factor (mNGF), which is widely used in the clinic, can influence the growth of interspinal schwannoma cells (ISCs) isolated from IS in vitro.

Methods : ISCs were isolated, cultured and identified by S-100 with immunofluorescence analysis. S-100-positive cells were divided into five groups, and separately cultured with various concentrations of mNGF (0 [phosphate buffered saline, PBS], 40, 80, 160 , and $320 \mathrm{ng} / \mathrm{mL}$ ) for 24 hours. Western blot and quantantive real time polymerase chain reaction (PCR) were applied to detect tyrosine kinase $A(T r k A)$ receptor and $p 75$ neurotrophin receptor $\left(p 75^{\text {NTR }}\right)$ in each group. Crystal violet staining was selected to assess the effect of $\mathrm{mNGF}(160 \mathrm{ng} / \mathrm{mL})$ on ISCs growth.

Results : ISCs growth was enhanced by mNGF in a dose-dependent manner. The result of crystal violet staining revealed that it was significantly strengthened the cells growth kinetics when cultured with $160 \mathrm{ng} / \mathrm{mL} \mathrm{mNGF}$ compared to PBS group. Western blot and quantantive real time PCR discovered that TrkA receptor and mRNA expression were both up-regualated under the condition of $\mathrm{mNGF}$, expecially in $160 \mathrm{ng} / \mathrm{mL}$, while the exoression of $\mathrm{p} 75^{\mathrm{NTR}}$ demonstrated no difference among groups.

Conclusion : From these data, we conclude that exogenous mNGF can facilitate ISC growth by activating both TrkA receptor and $\mathrm{p} 75^{\mathrm{NTR}}$. In addition, patients who are suffering from IS should not be administered mNGF in the clinic.

Key Words : Schwannoma $\cdot$ Mouse nerve growth factor $\cdot$ TrkA $\cdot$ p $75^{\text {NTR }}$.

- Received : March 27, 2019 • Revised : May 9, 2019 •Accepted : May 23, 2019

- Address for reprints : Shu Yi Liu

School of Clinical Medicine, Xi'an Medical University, 1 Xinwang Road, Xi'an, Shannxi 710021, China

Tel : +86-18829588435, Fax : +86-02986177489, E-mail : doclsy@163.com, ORCID : https://orcid.org/0000-0001-6495-5293

*These authors contributed equally to this work.

This is an Open Access article distributed under the terms of the Creative Commons Attribution Non-Commercial License (http://creativecommons.org/licenses/by-nc/4.0) which permits unrestricted non-commercial use, distribution, and reproduction in any medium, provided the original work is properly cited. 


\section{INTRODUCTION}

Nerve growth factor (NGF) is one of the most important growth factors in the nervous system and plays a crucial role in the regulation of neuronal development, the growth of axons, the synthesis of neurotransmitters, neuronal cell apoptosis, and inflammatory hyperalgesia ${ }^{22)}$. NGF is composed of $2 \alpha$ subunits, a $1 \beta$ subunit, $2 \gamma$ subunits and one or two zinc ions, and the $\beta$ subunit is the biologically active site ${ }^{28,31)}$. As a member of the neurotrophin family, NGF commonly exerts biological effects by binding to specific receptors on cell surfaces, including tyrosine kinase A (TrkA) and p75 neurotrophin receptors $\left(\mathrm{p} 75^{\mathrm{NTR}}\right)^{21)}$. TrkA has a high affinity for NGF, and activation of the NGF-TrkA signaling pathway can promote some cell proliferation, survival or metastasis ${ }^{8,9,12)}$. However, the $\mathrm{p} 75^{\mathrm{NTR}}$ receptor often exhibits a low affinity for NGF and is highly involved in cell apoptosis ${ }^{5,26)}$. Due to its homology with human NGF, mouse nerve growth factor (mNGF) is applied as a drug for the treatment of various central nervous system and peripheral system diseases in humans, especially for the repair of peripheral nerve injury and craniocerebral trauma ${ }^{30)}$.

Although many studies have indicated the important role of neurotrophin in the developing and mature nervous system, NGF was only recently revealed to play a role in the onset and progression of neurogenic and non-neurogenic tumors. For example, NGF can induce medulloblastoma cell apoptosis by modulating the activation of $\operatorname{Trk} \mathrm{A}^{3)}$. On the contrary, the overexpression of TrkA results in the promotion of breast cancer cell metastasis in immunodeficient mice ${ }^{15)}$. Furthermore, according to the reports of Khwaja et al. ${ }^{13)}$ and Krygier and Djakiew ${ }^{14)}$, the binding of NGF to $\mathrm{p} 5^{\mathrm{NTR}}$ can induce prostate tumor cell apoptosis. These observations suggest that there are large differences in the effects exerted by NGF among tumor cells, which may be dependent on the type of tumor cell line and may correspond to different transduction mechanisms of the NGF signaling pathway. Consequently, it is necessary to identify the roles of NGF in all types of tumors before considering applying $\mathrm{mNGF}$ to patients.

While some scholars have elucidated the effect of NGF on partial tumors in vivo and in vitro, its effect on interspinal schwannoma (IS) remains unclear. As the most common benign tumor of the spinal cord, IS generally originates from the sheath of dorsal nerve roots, an overwhelming majority of IS tumors are located in the subdural space and outside of the spinal cord ${ }^{11)}$. IS tumors are generally categorized into two pathological types : schwannoma and neurofibromas, which are composed of Schwann cells and fibroblasts respectively. According to information statistics, the incidence of IS is approximately $0.3-0.4 / 100000^{7)}$, and there are no epidemiological differences between males and females. The initial symptom of IS is spinal root pain, which is caused by the compression of tumors. Clinically, surgical resection under a microscope is the most common treatment for IS ${ }^{34)}$, although the procedure is frequently accompanied by complications ${ }^{37)}$, such as severe nerve root injury. Moreover, not all patients are suitable candidates for the surgical procedure. Although the pathological mechanisms of IS remain unclear, some researchers have implied an association of IS with NF1/NF2 gene mutations ${ }^{18)}$.

In the present study, we cultured primary interspinal schwannoma cells (ISCs) with various concentrations of mNGF to evaluate its effect in vitro. The data suggest that mNGF promotes ISC growth through the activation of TrkA receptors and $\mathrm{p} 75^{\mathrm{NTR}}$.

\section{MATERIALS AND METHODS}

\section{Primary culture of ISCs}

The study protocol was approved by the Medical Ethical Committee of Fuzhou Second Affiliated Hospital of Xiamen University, and consent was obtained from each patient involved in this study. Tumor tissue was acquired from patients who were definitively diagnosed to have IS based on preoperative magnetic resonance imaging (Fig. 1A) and postoperative pathological examinations (Fig. 1B-D). All patients underwent a microsurgical resection, and postoperative tumor spices were cautiously collected for the primary ISCs culture. ISCs were isolated according to a modified procedure by Zhang et al. ${ }^{35)}$. Briefly, specimens were transferred to a clean bench as quickly as possible, cut into pieces, plated in a petri dish, digested by $0.2 \%$ (wt/vol) collagenase I (Sigma) in DMEM (Invitrogen/GIBCO, Grand Island, NY, USA) for 12 hours, and incubated at $37^{\circ} \mathrm{C}$ and $100 \%$ humidity $/ 5 \% \mathrm{CO}_{2}$. After digestion, tissue fragments were transferred to another petri dish and pre-processed with a poly-lysine solution (Beyotime) and a laminin solution (Sigma), and the culture media was changed every 2-3 days. 

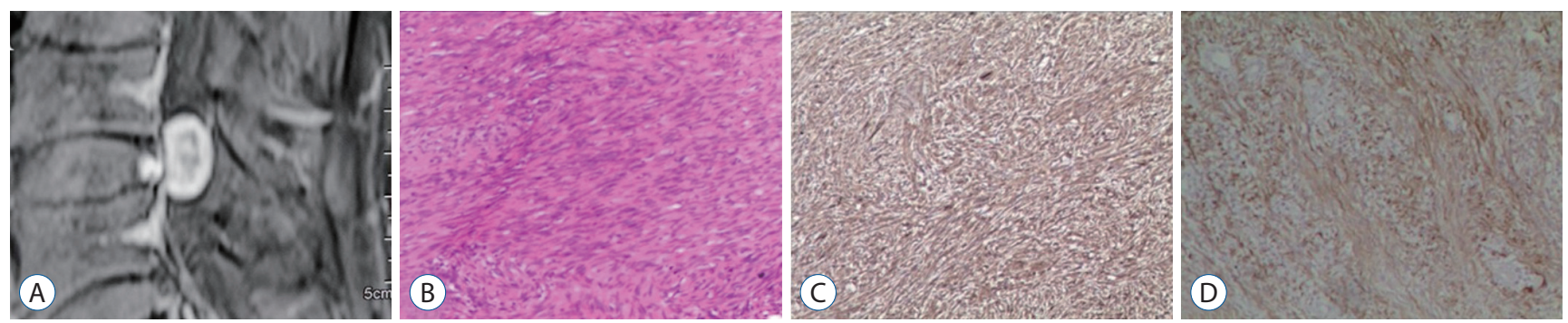

Fig. 1. MRI and pathological characteristics of IS. A : Contast enhanced sagittal T1A weighted MRI showing the tumor. B: Tumor tissue was stained by HE staining $(\times 100)$. C : Tumor tissue was stained for S-100 (brown; $\times 100)$. D : Tumor tissue was stained for GFAP (brown; $\times 100)$. MRI : magnetic resonance imaging, IS : interspinal schwannoma, HE : hematoxylin-eosin, GFAP : glial fbrillary acidic protein.

\section{Immunofluorescence}

ISCs were identified based on the protocol reported by Zhang et al. ${ }^{35}$. Briefly, ISC slides were prepared by fixing with $4 \%$ paraformaldehyde for an hour and washing the slides three times for 5 minutes each with phosphate buffered saline (PBS). Then, the cell membranes were permeabilized with $0.1 \%$ Triton X-100, and after 20 minutes, the cell slides were incubated with 7.5\% BSA at room temperature for 60 minutes and then washed with $1 \%$ bovine serum albumin (BSA). Subsequently, the slides were immersed in a monoclonal antiS-100 (Sigma, b subunit) solution $(1: 200)$ and placed in a humidified box and incubated overnight under continuous shaking at $4^{\circ} \mathrm{C}$. The slides were then incubated with fluorescein isothiocyanate anti-rabbit IgG (Beyotime, $1: 100$ ) at room temperature for 60 minutes. Finally, 4',6-diamidino-2-phenylindole (DAPI) was used to counterstain the nuclei, and the cells were observed images under an inverted microscope. To determine the cell purity, five non-overlapping horizons were randomly selected, and the DAPI-positive (total number of cells in each horizon) and S-100-positive cells were counted in each horizon under the inverted microscope. Therefore, the cell purity was indicated as the number of anti-S-100-positive cells divided by the number of DAPI-positive cells and multiplied by $100 \%$.

\section{Growth kinetics assay}

Crystal violet staining was selected to assess the effect of mNGF (Nobex; Sinobioway Medicine, Xiamen, China) on ISCs growth with the passage of time according to Chattopadhyay and Shubayev ${ }^{2}$. Briefly, the cells were plated in triplicate at $100 \mathrm{~mL}$ with 5000 cells/well in a 96-well plate and allowed to grow in complete medium with or without $160 \mathrm{ng} / \mathrm{mL}$ mNGF. then fixed with $1 \%$ glutaraldehyde for 20 minutes and stained by $0.1 \%$ crystal violet for 45 minutes at room temperature. Unbound dye was washed away with water, while bound dye was eluted with $10 \%$ acetic acid and the absorbance measured at $590 \mathrm{~nm}$. The value of relative increase of absorbance versus time \pm standard deviation (SD) was plotted for each time-point relative to absorbance values of cells attached overnight (representing a 0 hour time-point).

\section{Western blotting}

Twenty-four hours in advance, ISCs were plated in a 6-well plate at a density of $1 \times 10^{6}$ cells $/ \mathrm{mL}$ and were treated with the different concentrations of mNGF described above. Proteins were extracted using a lysis buffer (radio immunoprecipitation assay : $1 \%$ triton $\mathrm{X}-100,1 \%$ deoxycholate, $0.1 \%$ SDS; 1 $\mathrm{mM}$ PMSF). The total protein was measured using a BCA Protein Assay Kit (Beyotime). Samples containing equal amounts of protein $(10 \mu \mathrm{g})$ were run on a 10\% SDS-PAGE gel and then transferred to a PVDF membrane (Millipore, Billerica, MA, USA) in Tris-glycine transfer buffer (Beyotime) at $300 \mathrm{~mA}$ for 90 minutes. The membranes were blocked with $5 \%$ nonfat milk (Bio-Rad), incubated with a primary antibody (rabbit monoclonal anti-TrkA antibody (Abcam, cat. no. ab76291; Cambridge, MA, USA) and rabbit monoclonal antip75 ${ }^{\mathrm{NTR}}$ antibody (Abcam, cat. no. ab52987) in 5\% BSA in tris buffered saline (TBS) overnight at $4^{\circ} \mathrm{C}$, washed in TBS containing $0.05 \%(\mathrm{v} / \mathrm{v})$ Tween 20, and incubated for 1 hour at room temperature with an HRP-conjugated anti-rabbit (Abcam) or anti-mouse secondary antibody (Abcam). The blots were developed using enhanced chemiluminescence (Beyotime) and ChemiDoc ${ }^{\mathrm{TM}}$ XRS+ System with Image $\mathrm{Lab}^{\mathrm{TM}}$ Software (Bio-Rad Laboratories, Inc., Hercules, CA, USA). All blots represent at least three independent in vitro experiments. 
Table 1. The sequences of gene-specific primers

\begin{tabular}{|c|c|c|c|c|}
\hline Lot No. & Oligo name & Sequence $\left(5^{\prime}\right.$ to $\left.3^{\prime}\right)$ & Length & Purification \\
\hline 8405594803 & GAPDH (Ra)-F & ACG GCA AGT TCA ACG GCA CAG & 21 & HAP \\
\hline 8405594804 & GAPDH (Ra)-R & GAA GACGCC AGT AGA CTC CAC GAC & 24 & HAP \\
\hline 8405594787 & $\mathrm{p} 75^{\mathrm{NTR}}(\mathrm{hu})-\mathrm{F}$ & CAT CCT GGC TGC TGT GGT TGT & 21 & HAP \\
\hline 8405594788 & $\mathrm{p} 75^{\mathrm{NTR}}(\mathrm{hu})-\mathrm{R}$ & GTC TAT GTG CTC GGG CTG GTA & 21 & HAP \\
\hline 8405594789 & TrkA (hu)-F & TTC AAT GGC TCC GTG CTC AAT G & 22 & HAP \\
\hline 8405594790 & TrkA (hu)-R & GGT CTC CAG ATG TGC TGT TAG TGT & 24 & HAP \\
\hline
\end{tabular}

HAP : high affinity purification
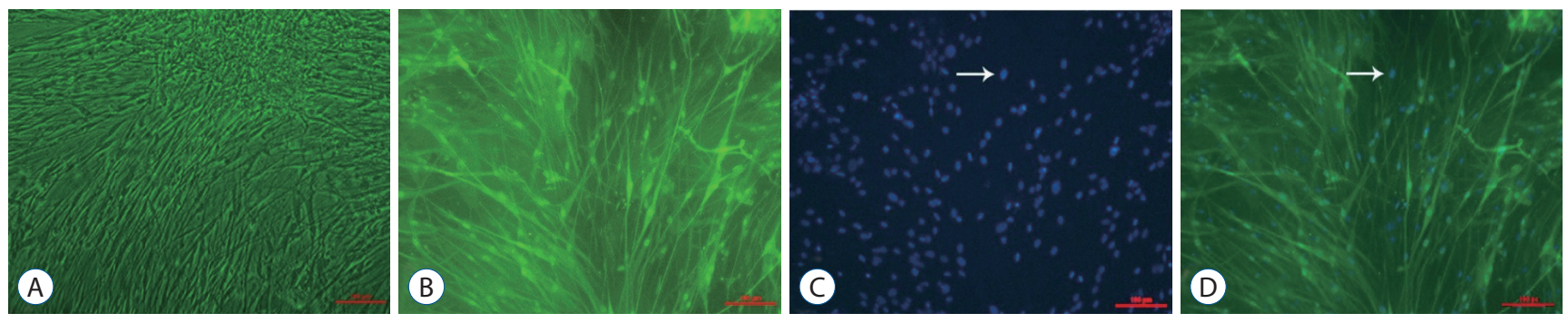

Fig. 2. Morphological characteristics of the primary cultured ISCs. A : ISCs were isolated from tumor tissue blocks after 48 hours of primary culture and demonstrated typical bipolar or tripolar morphologies (x100). B : ISCs were brightly stained for S-100 (green; ×100). C : ISCs and fibroblasts (white arrow) nuclei counterstained by DAPI (blue; $\times 100$ ). D : Merged image and fibroblasts were not stained for S-100 (white arrow). Scale bars, $100 \mu \mathrm{m}$. ISCs : interspinal schwannoma cells, DAPI : 4',6-diamidino-2-phenylindole, dihydrochloride.

\section{Quantitative real-time PCR}

Primers for human $\mathrm{p} 75^{\mathrm{NTR}}$ and TrkA were designed using Oligo 7.0 software (Table 1). ISCs were plated in 6-well plates at a density of $1 \times 10^{6}$ cells $/ \mathrm{mL}$. After 24 hours, the wells were treated with different concentrations of mNGF, as listed above, and continuously incubated for an additional 24 hours. The total RNA was extracted from each group of ISCs using an RNeasy ${ }^{\circledR}$ Mini Kit (Qiagen, Redwood, CA, USA). The RNA purity was verified by the OD260/280 absorption ratio of 2.0. cDNA was synthesized using a Revertaid ${ }^{\mathrm{TM}}$ First Strand cDNA Synthesis Kit (Thermo Scientific, Waltham, MA, USA). Gene expression was measured by quantitative real-time PCR using 50 ng cDNA and SYBR ${ }^{\oplus}$ Premix Ex Taq ${ }^{\mathrm{TM}}$ with the program (Takara Biomedical Technology [Beijing] Co., Ltd., Beijing, China) : $50^{\circ} \mathrm{C}$ for 2 minutes followed by $95^{\circ} \mathrm{C}$ for 10 minutes, $55^{\circ} \mathrm{C}$ for 15 seconds, and 40 cycles at $60^{\circ} \mathrm{C}$ for 30 seconds. Duplicate samples without cDNA (no-template control) verified no contaminating DNA, and every group corresponded to three repeated samples. Glyceraldehyde-3-phosphate dehydrogenase was used as a normalizing gene. The relative mRNA levels were quantified using the comparative cycle threshold method $^{15}$. Fold changes were determined using ABI 7500 Fast software (Applied Biosystems, Inc., Waltham, MA, USA) and the described methods.

\section{Statistical analysis}

The data are expressed as the means \pm SD based on at least three independent experiments. Differences among groups were determined via one-way analysis of variance, and comparisons between two groups were performed with Student's t-tests using SPSS 19.0 software (SPSS Inc., Chicago, IL, USA). $p<0.05$ was considered statistically significant.

\section{RESULTS}

\section{ISC culture and identification}

Isolated ISCs from the primary cultures displayed typical bipolar or tripolar morphologies based on phase-contrast microscopy (Fig. 2A). The Schwann cells that exhibited this unique morphology were positive for S-100, whereas the negative cells were fibroblasts (Fig. 2B); all of the cell nuclei stained positive for DAPI (Fig. 2C). The purity of the isolated Schwann cells was determined based on the combination of S-100 and 

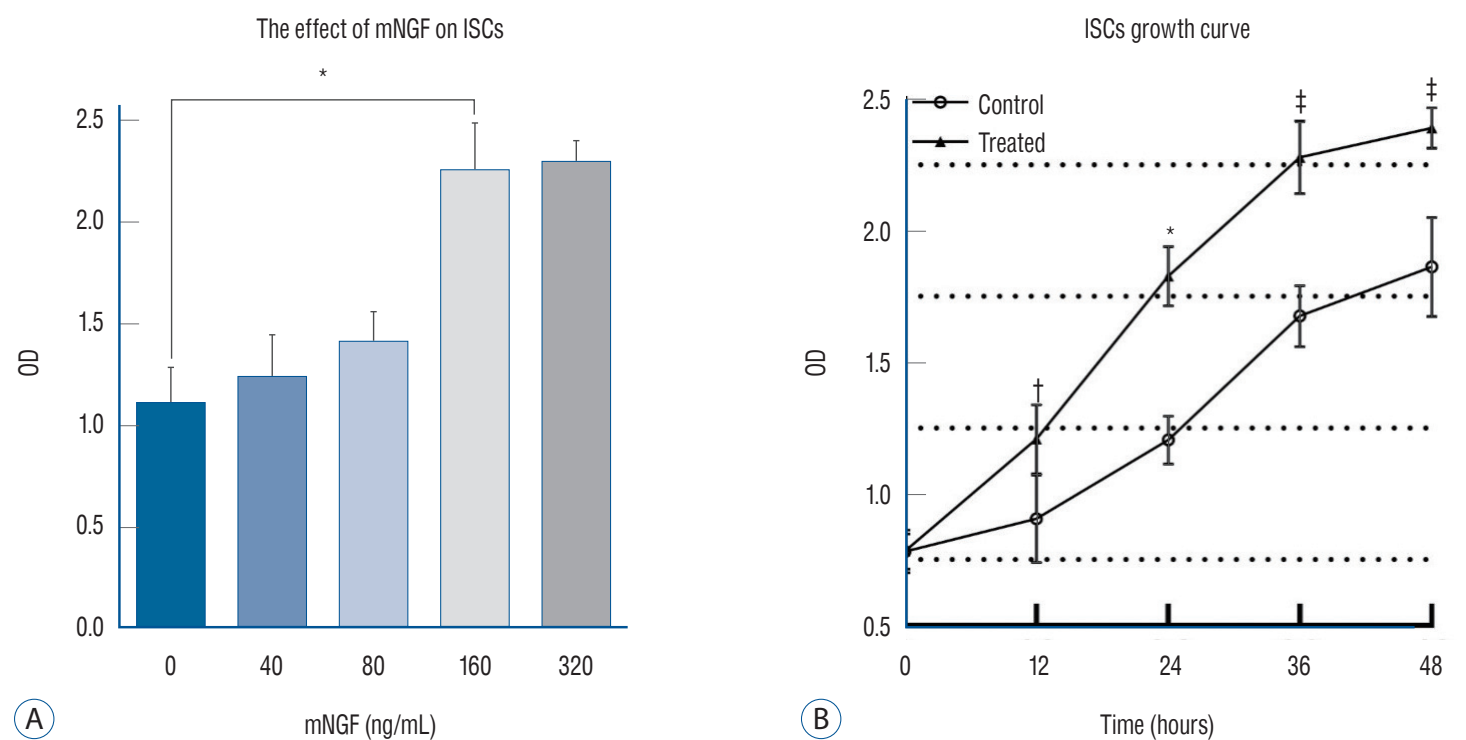

Fig. 3. mNGF can accelerate the growth of ISCs. A : The effect of different mNGF concentrations on ISCs was dose-dependent, and the peak effect was observed for the concentration of $160 \mathrm{ng} / \mathrm{mL}$. B : ISC growth curve studies using crystal violet with and without daily $160 \mathrm{ng} / \mathrm{mL} \mathrm{mNGF}$ stimulation for $12,24,36$, and 48 hours. The data represent the mean \pm standard error. ${ }^{*} p<0.001 .^{\dagger} p<0.05 .{ }^{\dagger} p<0.01$. mNGF : mouse nerve growth factor, ISCs : interspinal schwannoma cells.

DAPI immunofluorescence staining (Fig. 2D). Approximately $95.77 \pm 1.18 \%$ of the Schwann cells (DAPI-positive cells) were S100 -positive in the five randomly selected horizons.

\section{mNGF stimulation induces significant ISCs growth}

To identify the biological effect of mNGF on ISC proliferation, we generated a series of mNGF concentration gradients that included 40, 80, 160, and $320 \mathrm{ng} / \mathrm{mL}$, and PBS was used as a control. The same numbers of cells were incubated under the mNGF and PBS conditions, and then the cell counts were estimated using a CCK- 8 kit after at 24 hours. The optical density values gradually increased and ultimately reached a peak at the concentration of $160 \mathrm{ng} / \mathrm{mL}$ (Fig. 3A). Based on these data, the $160 \mathrm{ng} / \mathrm{mL}$ condition was selected for determining the ISC growth curve. Significantly enhanced ISC growth was observed after daily mNGF treatment over the course of 48 hours compared with the control group (Fig. 3B), suggesting that mNGF was correlated with ISC growth kinetics and that it induced a dose-dependent effect.

\section{The protein expression and mRNA level of TrkA and $\mathrm{p} 75^{\mathrm{NTR}}$ in ISCs}

Because NGF not only mediated cell proliferation but also enhanced apoptosis via its binding with TrkA and/or p75 receptors, the overall effect of NGF was generally based on the quantitative distributions of TrkA and/or p75 ${ }^{\mathrm{NTR}}$ receptors on the membranes of the cells. Therefore, we detected the expression of TrkA and $\mathrm{p} 75^{\mathrm{NTR}}$ when the ISCs were stimulated for 24 hours by the concentrations of mNGF listed above. The western blot results demonstrated that TrkA expression increased in parallel with the increasing concentrations of mNGF, especially at 160 and $320 \mathrm{ng} / \mathrm{mL}$, whereas $\mathrm{p} 75^{\mathrm{NTR}}$ expression was stable among the groups (Fig. 4). Consistent with this finding, the relative level of TrkA mRNA was prominently modulated by the different concentrations of mNGF compared with the control group, but the relative $\mathrm{p} 75^{\mathrm{NTR}}$ mRNA expression exhibited no significant changes among the groups (Fig. 5). All these data are consistent with the observed phenomenon of ISC growth under the conditions of different concentrations of mNGF.

\section{DISCUSSION}

These data are the first to imply a role of mNGF in regulating ISC proliferation, as its activation in the NGF-TrkA and NGF-p $75^{\text {NTR }}$ signaling pathway. Furthermore, we found that the acceleration of ISC growth was modulated by mNGF a dose-dependent manner. Unfortunately, the downstream mo- 

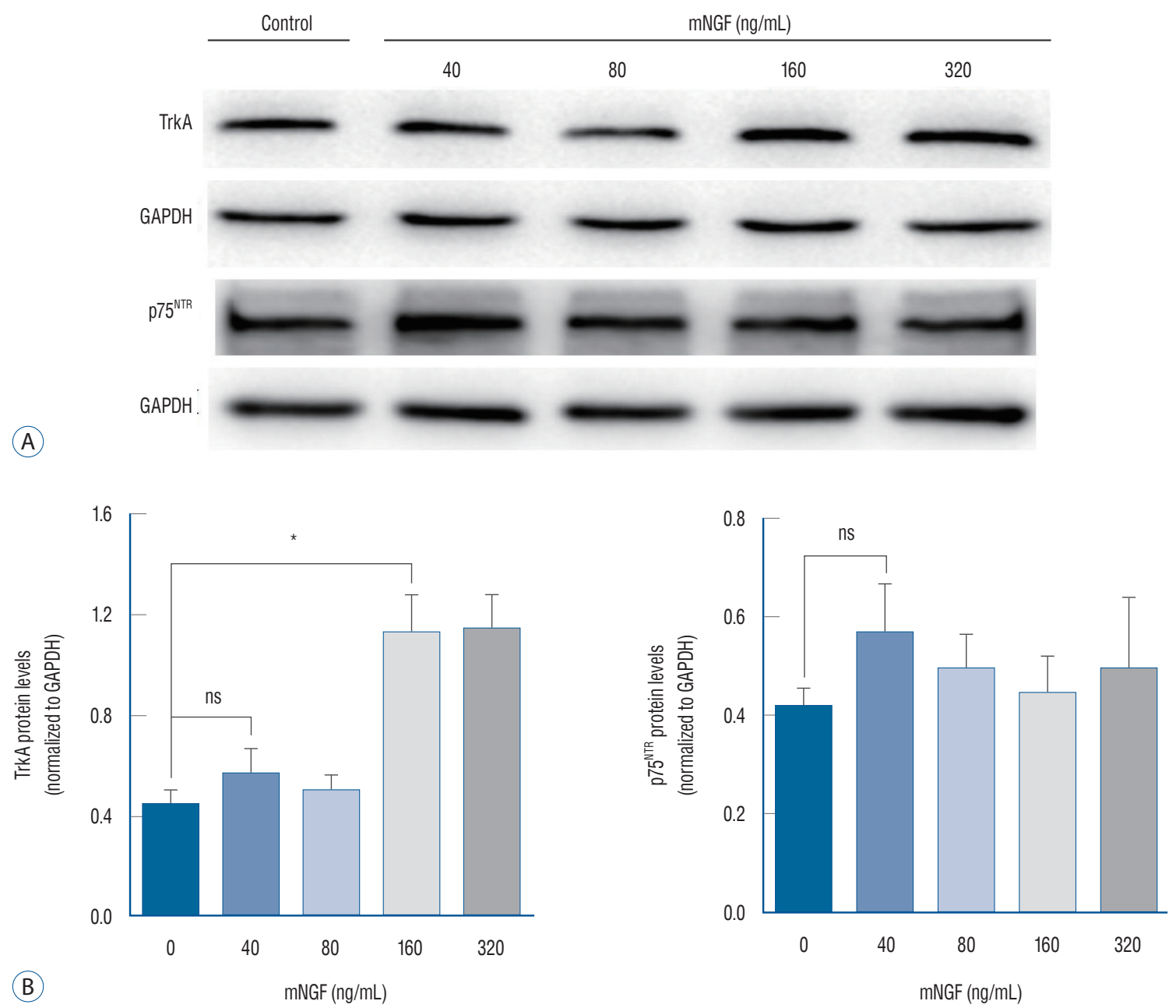

Fig. 4. mNGF can promote the expression of TrkA. A : Western blot for evaluating TrkA and p $75^{\text {NTR }}$ expression in ISCs at 24 hours. TrkA gradually increased with increasing concentrations of $\mathrm{mNGF}$, especially for the $160 \mathrm{ng} / \mathrm{mL}$ condition, compared with the control group (PBS), whereas the p75 $5^{\mathrm{NTR}}$ did not significantly change between the treated group and control group. B : The statistical analysis of the TrkA and p $75^{\mathrm{NTR}}$ level. The data represent the mean \pm standard error. ${ }^{*} p<0.01$. mNGF : mouse nerve growth factor, TrkA : tyrosine kinase A, GAPDH : glyceraldehyde-3-phosphate dehydrogenase, $\mathrm{p} 75^{\mathrm{NTR}}$ : p75 neurotrophin receptor, ns : nonsignificant, PBS : phosphate buffered saline.

lecular mechanisms of NGF-TrkA and NGF-p75 ${ }^{\text {NTR }}$ signaling have yet to be fully elucidated. Recent studies have explored the role of NGF in tumor induction and progression. In human pancreatic cancer, NGF can mediate cell growth in relation to the expression levels and ratio of TrkA and p75 ${ }^{\text {NTR 36). }}$ George et al. ${ }^{6}$ concluded that genetic mutations of TrkA do not seem to play a significant role in the activation of this pathway in prostate cancer but found an absence of mutations in otherwise genetically unstable prostate tumor DNA, which suggests that intact NGF-TrkA pathways may be important in prostate cancer development. Lagadec et al. ${ }^{15)}$ provided the first direct evidence that TrkA overexpression enhances the tumorigenic properties of breast cancer cells. Moreover, Wad- hwa et al. ${ }^{32}$ indicated that TrkA receptors are involved in tumor pathogenesis and in medullary thyroid carcinoma ${ }^{19)}$ and that they contribute to the progression of tumors toward malignancy. Accumulating data have demonstrated that the NGF-TrkA signaling pathway also participates in the growth and invasion of lung cancer cells $\mathrm{s}^{25}$ and ovarian carcinomas ${ }^{4)}$. All these studies highlight the hypothesis that NGF and TrkA play vital roles in tumor cell development and progression. However, some researchers have also reported contrasting findings regarding the roles of NGF and TrkA in other types of tumors. For example, in neuroblastomas, high levels of TrkA expression were a sign of favorable prognosis and tended to spontaneously decrease ${ }^{24}$. Therefore, NGF and TrkA may 
Relative expression of TrkA in ISCs

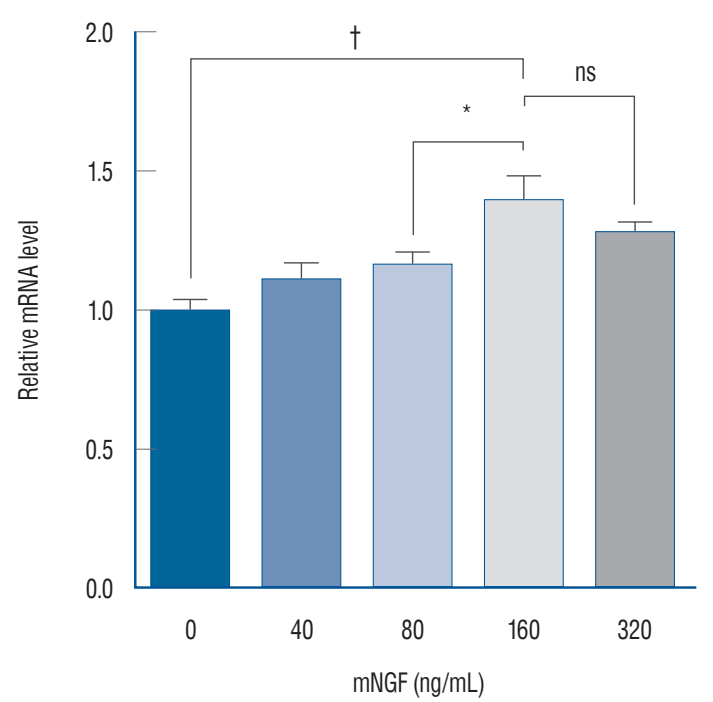

Relative expression of $\mathrm{p} 75^{\mathrm{NTR}}$ in ISCS

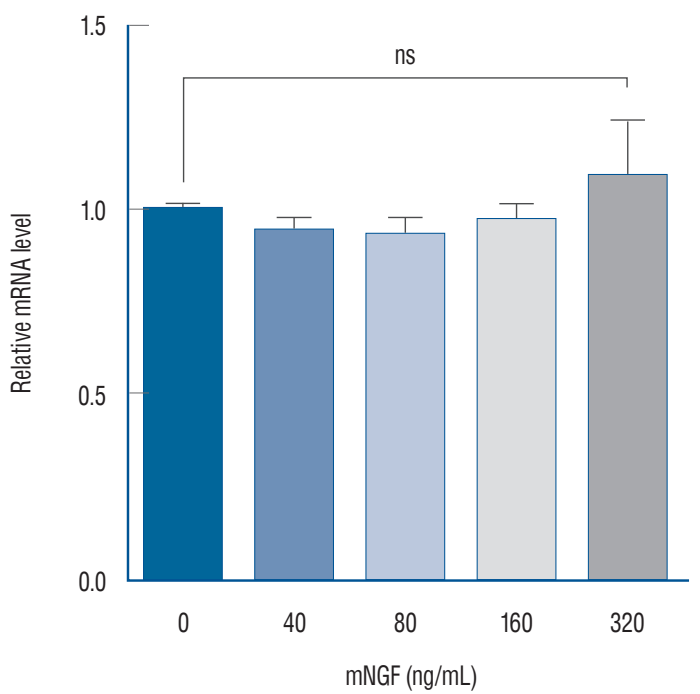

Fig. 5. Real-time TaqMan qPCR for the mRNA expression levels in the ISCs at 24 hours. Each group normalized to GAPDH and calibrated to the sham for increasing mNGF concentrations. The mRNA levels of TrkA were significantly increased for the $160 \mathrm{ng} / \mathrm{mL}$ condition compared to the control group (PBS), but the mRNA levels of $p 75^{N T R}$ did not significantly differ among groups, which were consistent with the results of the western blot. The data represent the mean \pm standard error. ${ }^{*} p<0.05 .{ }^{\dagger} p<0.01$. TrkA : tyrosine kinase A, ISC : interspinal schwannoma cell, $\mathrm{p} 75^{\mathrm{NTR}}$ : p75 neurotrophin receptor, ns : nonsignificant, qPCR : quantitative polymerase chain reaction, mRNA : messenger-ribonucleic acid, GAPDH : glyceraldehyde-3-phosphate dehydrogenase, mNGF : mouse nerve growth factor, PBS : phosphate buffered saline.

play a variety of roles in different types of tumors.

p $75^{\mathrm{NTR}}$ belongs to the tumor necrosis factor superfamily and is commonly associated with the induction of cell apoptosis ${ }^{27}$, although recent studies have revealed greater effects of the activation of the NGF-p $75^{\text {NTR }}$ pathway. On the one hand, NGF binding to $\mathrm{p} 75^{\mathrm{NTR}}$ can activate NF- $\kappa \mathrm{B}$ signaling involving BEX2, which has been definitively shown to promote tumor cell survival ${ }^{1}$; on the other hand, in cells that co-express p $75^{\text {NTR }}$ and TrkA, p75 ${ }^{\text {NTR }}$ not only prolongs cell-surface TrkAdependent signaling events by negatively regulates receptor ubiquitination but is also capable of regulating TrkA receptor binding properties via the neurotrophin receptor homolog-2, a transmembrane protein related to $\mathrm{p} 75^{\mathrm{NTR} 1723)}$. Thus, the effect of NGF on tumors also depends on the expression levels of TrkA and $\mathrm{p} 75^{\mathrm{NTR}}$ within the tumor cells.

Although significant efforts had been made to explore the roles of NGF in tumors, its effects on IS are not fully resolved. Furthermore, due to its benign nature, it is often ignored in studies. In our study, ISCs were isolated from the tumor tissue and treated with a series of mNGF concentrations. Interestingly, the cells proliferated well and exhibited TrkA expression and relative mRNA levels that gradually increased compared to the control group. In particular, these increases were highly significant for the $160 \mathrm{ng} / \mathrm{mL}$ treatment condition. Subsequently, we stimulated ISCs for 12, 24, 36, and 48 hours with $160 \mathrm{ng} / \mathrm{mL}$ mNGF or PBS to generate the ISC growth curve. We found that cell growth was strongly enhanced at every time point compared with the control group. However, $\mathrm{p} 75^{\mathrm{NTR}}$ expression and its relative mRNA levels remained constant among all groups. These data suggest that mNGF can markedly promote ISC growth in vitro by activating the NGF-TrkA and NGF-p $75^{\text {NTR }}$ signaling pathways. Moreover, the activation of p $75^{\text {NTR }}$ in ISCs causes the exposure of high-affinity binding sites on NGF, thus improving the binding rate of NGF with TrkA but not inducing cell apoptosis.

mNGF (Enjingfu) is manufactured as a neurotrophic drug by Sinobioway Biomedicine Co., Ltd. (Xiamen, China) and is widely used in therapies for both central and peripheral nerve system diseases, such as spinal injury, traumatic brain injury, diabetic peripheral neuropathy, and traumatic peripheral injury. A recent study demonstrated that radiation-induced temporal lobe necrosis was successfully treated with $\mathrm{mNGF}^{33)}$. However, our results showed that mNGF promoted the growth of ISC in vitro, which suggests that it is not appropriate for use in situations where 1) IS is so complex that it cannot be completely resected, such as in dumbbell-type tumors; 
2) the size of the tumor is generally small, and the patient has no clinical symptoms; 3 ) the patient has another severe diseases and cannot tolerate operation; 4) the tumor is located in the $\mathrm{C}_{1-2}$ segment, wrapping the vertebral artery and rendering it unsuitable for operation; or 5) the IS is malignant. Therefore, it is recommended to avoid treating patients who are diagnosed with IS with mNGF or similar drugs. Moreover, base expression levels of TrkA and p $75^{\mathrm{NTR}}$ were detected in the control ISCs, which may highlight that a limited amount of NGF, primarily secreted by adjacent nerve fibers or other healthy tissue, exists in the microenvironment of IS to maintain cell survival. In breast cancer and oesophageal squamous cell carcinoma, NGF and TrkA frequently shows overexpression and a autocrine loop of NGF is also observed, which results in a low survival rate of patients ${ }^{10,29)}$. Therefore, abolishing the NGF-TrkA signaling pathway with a Trk tyrosine kinase inhibitor, such as CEP-70120), could be a potential approach for tumor therapy in the future.

\section{CONCLUSION}

NGF is indispensable for normal organisms, but its effects in some diseases are unpredictable, especially in tumors. In our study, we find that exogenous mNGF markedly stimulated the growth of ISCs and increased the expression of TrkA. Although there was no difference among groups, p75 ${ }^{\mathrm{NTR}}$ still had a little expression and may play a subsidiary role of TrkA. According to these phenomenon, it may be prudent to administer mNGF for clinicians. However, the conclusion is based on in vitro experiments, therefore, in vivo research will be lauched to reveal the effect of mNGF on IS patients and indicate its cinical application.

\section{CONFLICTS OF INTEREST}

No potential conflict of interest relevant to this article was reported

\section{INFORMED CONSENT}

Informed consent was obtained from all individual partici- pants included in this study.

\section{AUTHOR CONTRIBUTIONS}

\author{
Conceptualization : LSY, LSZ \\ Data curation : CS, LSY \\ Formal analysis : LY, CS \\ Funding acquisition : LSY, LSZ \\ Methodology : LSY \\ Project administration : LSZ \\ Visualization : LSY \\ Writing - original draft : LSY, LY \\ Writing - review \& editing : LSZ, LSY, LY, CS
}

\section{References}

1. Ahmad I, Yue WY, Fernando A, Clark JJ, Woodson EA, Hansen MR : p75NTR is highly expressed in vestibular schwannomas and promotes cell survival by activating nuclear transcription factor kappa B. Glia 62 : 1699-1712, 2014

2. Chattopadhyay S, Shubayev VI : MMP-9 controls Schwann cell proliferation and phenotypic remodeling via IGF-1 and ErbB receptor-mediated activation of MEK/ERK pathway. Glia 57 : 1316-1325, 2009

3. Chou TT, Trojanowski JQ, Lee VM : A novel apoptotic pathway induced by nerve growth factor-mediated TrkA activation in medulloblastoma. J Biol Chem 275 : 565-570, 2000

4. Davidson B, Reich R, Lazarovici P, Nesland JM, Skrede M, Risberg B, et al. : Expression and activation of the nerve growth factor receptor TrkA in serous ovarian carcinoma. Clin Cancer Res 9 : 2248-2259, 2003

5. Friedman WJ : Proneurotrophins, seizures, and neuronal apoptosis. Neuroscientist 16 : 244-252, 2010

6. George DJ, Suzuki H, Bova GS, Isaacs JT : Mutational analysis of the TrkA gene in prostate cancer. Prostate 36 : 172-180, 1998

7. Gu R, Liu JB, Zhang Q, Liu GY, Zhu QS : MRI diagnosis of intradural extramedullary tumors. J Cancer Res Ther 10 : 927-931, 2014

8. Haase G, Pettmann B, Raoul C, Henderson CE : Signaling by death receptors in the nervous system. Curr Opin Neurobiol 18 : 284-291, 2008

9. Hellebrand $\mathrm{EE}$, Varbiro $\mathrm{G}$ : Development of mitochondrial permeability transition inhibitory agents: a novel drug target. Drug Discov Ther 4 : 54-61, 2010

10. Hondermarck $\mathrm{H}$ : Neurotrophins and their receptors in breast cancer. Cytokine Growth Factor Rev 23 : 357-365, 2012

11. Jeon JH, Hwang HS, Jeong JH, Park SH, Moon JG, Kim CH : Spinal schwannoma; analysis of 40 cases. J Korean Neurosurg Soc 43 : 135-138, 2008 
12. Johnson TV, Bull ND, Martin KR : Neurotrophic factor delivery as a protective treatment for glaucoma. Exp Eye Res 93 : 196-203, 2011

13. Khwaja F, Tabassum A, Allen J, Djakiew D : The p75(NTR) tumor suppressor induces cell cycle arrest facilitating caspase mediated apoptosis in prostate tumor cells. Biochem Biophys Res Commun 341 : 11841192, 2006

14. Krygier $S$, Djakiew D : The neurotrophin receptor p75NTR is a tumor suppressor in human prostate cancer. Anticancer Res 21 : 3749-3755, 2001

15. Lagadec C, Meignan S, Adriaenssens E, Foveau B, Vanhecke E, Romon R, et al. : TrkA overexpression enhances growth and metastasis of breast cancer cells. Oncogene 28 : 1960-1970, 2009

16. Livak KJ, Schmittgen TD : Analysis of relative gene expression data using real-time quantitative PCR and the $2^{-\triangle \Delta C T}$ method. Methods $25: 402$ 408, 2001

17. Makkerh JP, Ceni C, Auld DS, Vaillancourt F, Dorval G, Barker PA : p75 neurotrophin receptor reduces ligand-induced Trk receptor ubiquitination and delays Trk receptor internalization and degradation. EMBO Rep 6 : 936-941, 2005

18. Martinez-Glez V, Franco-Hernandez C, Alvarez L, de Campos JM., Isla A, Vaquero J, et al. : Meningiomas and schwannomas: molecular subgroup classification found by expression arrays. Int J Oncol 34 : 493-504, 2009

19. McGregor LM, McCune BK, Graff JR, McDowell PR, Romans KE, Yancopoulos $\mathrm{GD}$, et al. : Roles of trk family neurotrophin receptors in medullary thyroid carcinoma development and progression. Proc Natl Acad Sci U S A 96 : 4540-4545, 1999

20. Miknyoczki SJ, Dionne CA, Klein-Szanto AJ, Ruggeri BA : The novel Trk receptor tyrosine kinase inhibitor CEP-701 (KT-5555) exhibits antitumor efficacy against human pancreatic carcinoma (Panc1) xenograft growth and in vivo invasiveness. Ann N Y Acad Sci 880 : 252-262, 1999

21. Mitsiadis TA, Pagella $P$ : Expression of nerve growth factor (NGF), TrkA, and p75(NTR) in developing human fetal teeth. Front Physiol $7: 338$, 2016

22. Mizumura K, Murase $S$ : Role of nerve growth factor in pain. Handb Exp Pharmacol 227 : 57-77, 2015

23. Murray SS, Perez P, Lee R, Hempstead BL, Chao MV : A novel p75 neurotrophin receptor-related protein, $\mathrm{NRH2}$, regulates nerve growth factor binding to the TrkA receptor. J Neurosci 24 : 2742- 2749, 2004

24. Nakagawara A, Arima-Nakagawara M, Scavarda NJ, Azar CG, Cantor $A B$, Brodeur $G M$ : Association between high levels of expression of the
TRK gene and favorable outcome in human neuroblastoma. $\mathbf{N}$ Engl J Med $328: 847-854,1993$

25. Ricci A, Greco S, Mariotta S, Felici L, Bronzetti E, Cavazzana A, et al. : Neurotrophins and neurotrophin receptors in human lung cancer. Am J Respir Cell Mol Biol 25 : 439-446, 2001

26. Salehi $A H$, Xanthoudakis S, Barker PA : NRAGE, a p75 neurotrophin receptor-interacting protein, induces caspase activation and cell death through a JNK-dependent mitochondrial pathway. J Biol Chem 277 : 48043-48050, 2002

27. Skaper SD : The neurotrophin family of neurotrophic factors: an overview. Methods Mol Biol 846 : 1-12, 2012

28. Thoenen $H$, Barde YA : Physiology of nerve growth factor. Physiol Rev $60: 1284-1335,1980$

29. Tsunoda S, Okumura T, Ito T, Mori Y, Soma T, Watanabe G, et al. : Significance of nerve growth factor overexpression and its autocrine loop in oesophagealsquamous cell carcinoma. Br J Cancer 95 : 322-330, 2006

30. Ullrich A, Gray A, Berman C, Dull TJ : Human $\beta$-nerve growth factor gene sequence highly homologous to that of mouse. Nature 303 : $821-825,1983$

31. Varon S, Nomura J, Shooter EM : The isolation of the mouse nerve growth factor protein in a high molecular weight form. Biochemistry 6 : 2202-2209, 1967

32 Wadhwa S, Nag TC, Jindal A, Kushwaha R, Mahapatra AK, Sarkar C : Expression of the neurotrophin receptors Trk $A$ and Trk $B$ in adult human astrocytoma and glioblastoma. J Biosci 28 : 181-188, 2003

33. Wang $X$, Ying $H$, Zhou Z, Hu C, Eisbruch A : Successful treatment of radiation-induced temporal lobe necrosis with mouse nerve growth factor. J Clin Oncol 29 : e166-e168, 2011

34. Yang T, Wu L, Deng X, Yang C, Xu Y : Clinical features and surgical outcomes of intramedullary schwannomas. Acta Neurochir (Wien) 156 : 1789-1797, 2014

35. Zhang K, Chen S, Lin J, Zhang Y, Yu W, Liu S, et al. : Primary culture of human interspinal schwannoma. J Biomater Tissue Eng 6 : 263-269, 2016

36. Zhu ZW, Friess H, Wang L, Bogardus T, Korc M, Kleeff J, et al. : Nerve growth factor exerts differential effects on the growth of human pancreatic cancer cells. Clin Cancer Res 7 : 105-112, 2001

37. Zong S, Zeng G, Xiong C, Wei B : Treatment results in the differential surgery of intradural extramedullary schwannoma of 110 cases. PLoS One 8 : e63867, 2013 